\title{
Oral care in facilities for disabled people: interest of teledentistry
}

\author{
Roy Olivier ${ }^{1}$, Dodin Thibault ${ }^{2}$, Venuat Stéphane ${ }^{3}$, Dagregorio Aline ${ }^{3}$, Inquimbert Camille In $^{1,2,4}$, Valcarcel Jean ${ }^{1,2}$ and Giraudeau Nicolas ${ }^{1,2,5,6 *}$ \\ ${ }^{1}$ Public health and telemedicine department, dental department, University hospital of Montpellier, 547, Avenue du Pr Jean-Louis Vialla 34080 Montpellier, \\ France Montpellier, France \\ ${ }^{2}$ Dental Faculty, University of Montpellier, 545, Avenue du Pr Jean-Louis Viala 34080 Montpellier, France \\ ${ }^{3}$ FAM Le Guilhem, UNAPEI 34, 1804 Avenue du Père Soulas 34090 Montpellier, France \\ ${ }^{4}$ Laboratoire Parcours de santé systémique EA 4129 Université Claude Bernard Lyon 1 \\ ${ }^{5}$ e-Health Chaire, Foundation of University of Montpellier, 163, Avenue August Broussonnet 34967 Montpellier, France \\ ${ }^{6} \mathrm{CEPEL}$, Université de Montpellier, CNRS, Montpellier, France
}

\begin{abstract}
Oral health in facilities for disabled people is a real issue. Even if recommendations are proposed by national health agencies, it is still a fairly neglected area. A facility in Montpellier, South of France, has been working for several years to improve patients' oral health and a caregiver is specially involved in this aspect. This article is about evaluating the activity of this facility on oral health and identifying the opportunity to use teledentistry. We realized a retrospective analyse of patient medical files for a period of 58 months. We identify that, among the 415 appointments to the dentist, only $3 \%$ have been performed under general anaesthesia. Teledentistry could decrease this number by $25 \%$. Rather than saving money and time to the facility, teledentistry could also save energy and time from the patients and it is priceless. Teledentistry should be implemented to be officially evaluated in this type of facility, but it seems to be a good public health tool, on the theoretical aspect.
\end{abstract}

\section{Introduction}

"Any disabled person has the right to benefit of the entire solidarity of the national community which guarantees him, by virtue of this national obligation, access to fundamental care recognized to all citizens and the full exercise of citizenship". This sentence come from the law about the rights and chance equality, the involvement and the citizenship of disabled people enforced on February $11^{\text {th }} 2005$. However, the reality is completely different. Only few studies are developed on this subject and especially on oral care but they all show that there is a public health issue on the oral health condition of people having disability. For example, Dorin et al. present that only 3,4\% out of 8,401 children and teenagers with disability had no oral health disease [1]. In general, it is unfortunately admitted that the oral health status for people with disability is deteriorating along their age and is much poorer than for the rest of the population $[2,3]$. Number of recent international studies pointed the low status of dental care with more untreated dental situations and fewer basic oral hygiene [4-7]. This specific group is identified as showing a high level of risk of dental diseases [8]. In addition to the "traditional" risk factors, others could be referenced: medication, neuro-motor and sensitive incapacities, co-morbidity and opposition behaviour. These different risk factors impact the capacity for a caregiver to help or to realize the oral hygiene and of course to get the oral treatment more complicated. The possible non-cooperate patient's behaviours in same case of disabilities must impose us more preventive attitude to avoid oral health degradation.

As mentioned earlier, the oral health status of disabled people is a public health issue. Obviously, it is not new. French policy makers and stakeholders already proposed several actions to improve this situation. An oral consultation is recommended when the patient is entering the facility and then once a year. Unfortunately, this initial oral consultation is rarely carried out because of several conditions: maybe at first the lack of knowledge on oral health from the health professionals in the facilities for disabled people. We could also point out the difficulties for a dentist to go to this type of facility, and the complications for a patient and the medical staff to manage a transportation to the dental office as well.

Conscient of this critical situation, the university hospital of Montpellier developed in 2014 a teledentistry project named e-DENT $[9,10]$. The concept is to perform an oral diagnosis remotely. In the e-DENT project a caregiver or a nurse, who have been trained to dental knowledge and teledentistry, record information needed by the "teledentist" to realize a diagnosis. This information is:

- Medical data (medicine prescription, chronic diseases linked with oral health, ...)

- Dental history

- Videos showing the mouth of the patient recorded with an intra-oral camera using a specific light to have a relevant diagnosis through images analyse.

${ }^{*}$ Correspondence to: Giraudeau Nicolas, Public health and telemedicine department, dental department, University hospital of Montpellier, 547, Avenue du Pr Jean-Louis Vialla 34080 Montpellier, France Montpellier, France, E-mail: nicolas.giraudeau@umontpellier.fr

Key words: telemedicine, disabled persons, oral health

Received: May 20, 2019; Accepted: June 10, 2019; Published: June 13, 2019 
The "teledentist" can analyse the data and propose a diagnosis, a treatment plan and a specific medical pathway adapted to the patient, considering his cognitive, general and oral status.

This project was originally supported by the regional public health agency and only 16 nursing homes or facilities for disabled people were involved but, at the end of 2018, 30 facilities are able to benefit of the teledentistry project in the Occitanie region.

UNAPEI, an association of disabled people, asked for being integrated in the project. The university hospital of Montpellier launched a study to evaluate the oral health activity in a facility of Montpellier. They chose one which was very interested in the subject of oral health, aiming to identify how much this activity would cost and how teledentistry could be useful even in this type of expert facility.

\section{Materials and methods}

\section{Aims}

Principal: Evaluate the oral health activity in one facility for disabled people in France.

Secondary: Evaluate the cost of this activity. Study the opportunity to use a teledentistry system.

\section{Ethics statement}

This study was reviewed and approved by the regional ethical committee of Sud-Mediterranée III under the number 2014-A0003938. The study was conducted according to the principles of the Helsinki Declaration.

The consent of the people in charge of the patient has been collected before using their data.

\section{Participants}

The facility is one of those managed by the UNAPEI Association in Montpellier. They are in charge of 41 persons full time and 1 part time. They all have a mental disability ranked from middle to high. In this facility there is A caregiver especially in charge of oral care and eyes care is attached to this facility. He is dedicated full time to these two subjects.

\section{Study design}

This was a 5-year retrospective cross-sectional study on the medical files and activity on oral health in one facility for disabled people, from 2011, January $12^{\text {th }}$ to 2015 , October $5^{\text {th }}$ (58 months).

Files analyse: Every file has been anonymised by the caregiver in charge of the oral health. He attributed a code for each patient before giving us the files (e.g. A1, B3, F5...). These documents recorded all information about oral health status and actions made on this subject (e.g. which dentist has been involved, appointment dates, dental treatment reports, type of medical pathway).

Interest of teledentistry: We evaluated the number of appointments that could have been performed remotely through a teledentistry system. We defined an appointment which could be avoided thanks to teledentistry as one during no treatment has been realized on the patient

Financial: We proceeded with a cost study for the oral care involvement in this facility and how much money could be saved by using teledentistry.

We evaluated the time consumed by the caregiver when going to a private dentist or to the public hospital with a patient. Were included in that time: patient preparation, travel to the dentist, time during the treatment and the way back to the facility. We didn't include appointments for a general anaesthesia because this time could be very variable.

\section{Results}

\section{Population description}

38 people accepted to participate in this study. 4 consents were never returned by the patient's tutor.

\section{Age and sex}

The age average was 41,4 years old ( 24 till 60 ) and the facility was in charge of 22 male patients (58\%).

\section{Disability status}

Figures 1 and 2.

\section{Appointments}

General: A total of 415 appointments to a dentist have been collected. The averages are: 10,9 appointment by patient and 7,1 appointments per month.

$79 \%$ (327) of these appointments have been realized in a private practice and $21 \%(88)$ in the dental department of the university hospital. Out of these 88 appointments in the hospital, 77 (87\%) have been realized with conscious patients and the last 11 under general anaesthesia.

So, on the 415 appointments only $3 \%$ [11] have been done under general anaesthesia.

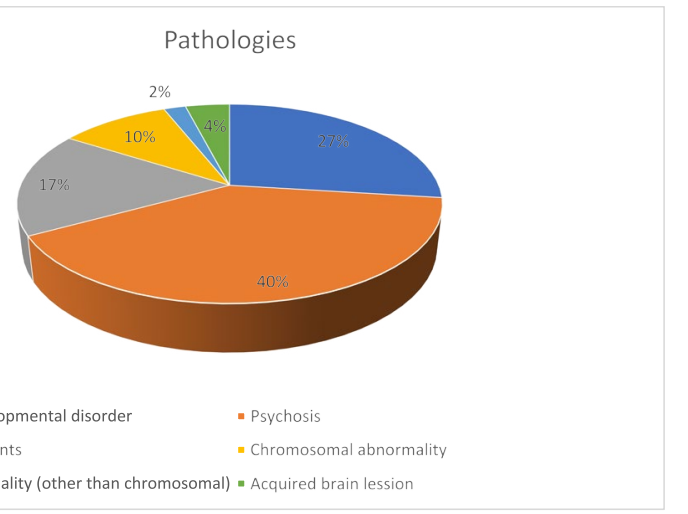

Figure 1. Pathologies distribution Disabilities

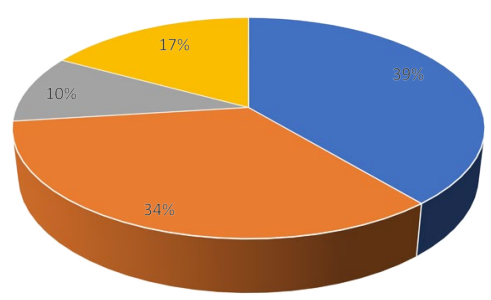

- Profound and severe mental retardation - Moderate mental retardation " Behaviour and conduct disorder $\quad$-Serious psychiatric problems

Figure 2. Disabilities distribution 


\section{Treatments}

Figure 3.

Out of the 34 dental extractions, 13 (38\%) have been done on patients under general anaesthesia and $21(62 \%)$ with conscious ones.

Out of the 21 prothesis, $11(52 \%)$ were removable and $10(48 \%)$ were crowns or bridges.

\section{Teledentistry}

We defined 104 appointments that teledentistry could had certainly avoided i.e. $25 \%$.

Out of these 104 appointments, 28 (27\%) have been organized in the university hospital of Montpellier and $76(73 \%)$ in the private practice (Figures 4 and 5).

\section{Financial}

415 appointments were fixed: 327 in private office and 77 (88 less 11 with general anaesthesia) in public hospital.

The average time for an appointment in private office is 75 minutes ( 1,25 hours) and the average time within a public hospital is 90 minutes (1,5 hours).

The caregiver cost for the facility is $18,51 €$ per hour (all taxes included).

Table 1.

We mentioned just above that we could have saved 104 appointments with teledentistry: 28 in public hospital and 76 in private practice.

Table 2.

\section{Discussion}

First of all, it is very challenging to find out in France a facility like this one, where a caregiver is clearly identified to take care of patients' oral health. The most frequent case in this type of facilities is not to have access to oral health [11].

Even if a lot of actions are proposed to improve oral health, the patients still need to be treated by the dentist. 7 appointments per month is a quite important number and may consume a lot of time for people in charge.

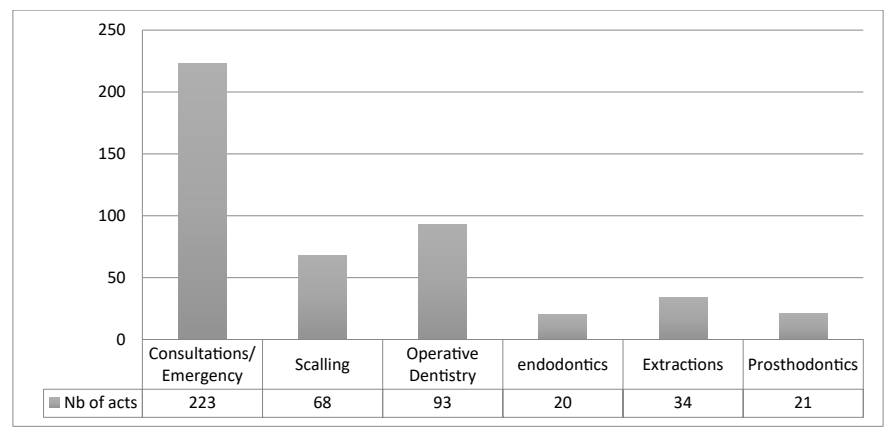

Figure 3. Treatments distribution

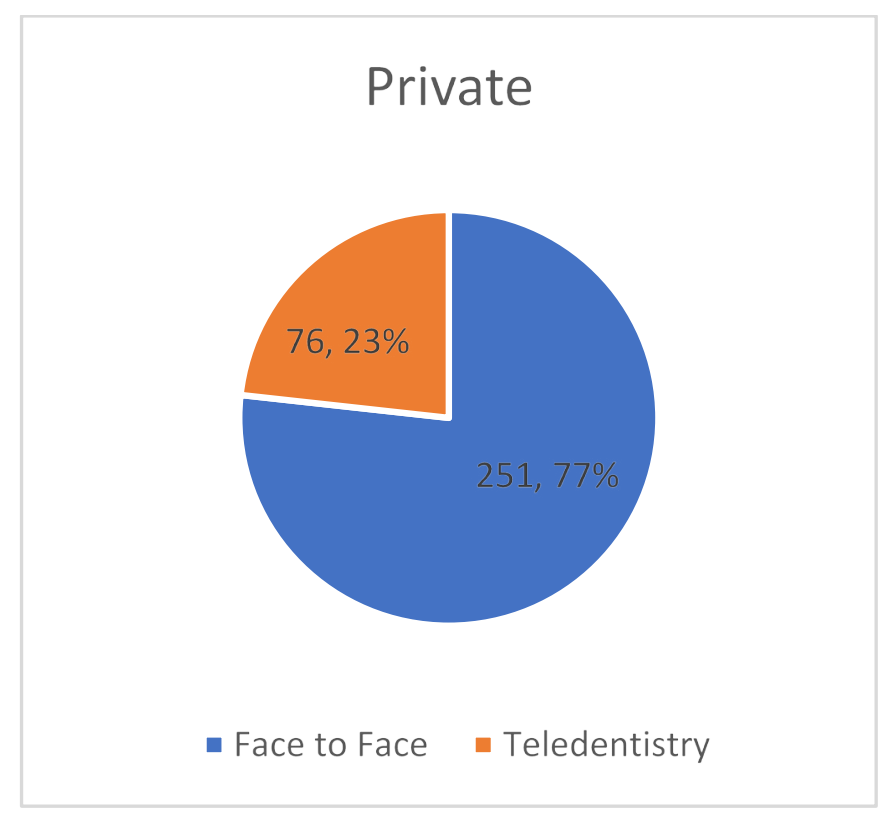

Figure 4. opportunity for teledentistry for appointment realized in private practice

\section{Public}

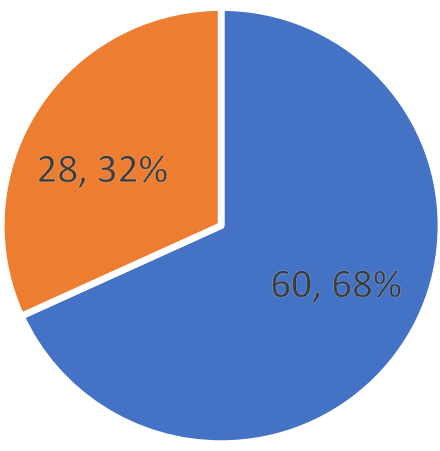

\section{- Face to Face - Teledentistry}

Figure 5. Opportunity for teledentistry for appointments realized in public hospital

Table 1. Cost of time dedicated to oral health during 58 months

\begin{tabular}{|c|c|c|c|}
\hline & By appointment & For all appointments & By month \\
\hline Private practice & $23,14 €$ & $7565,96 €$ & $130,45 €$ \\
\hline Public Hospital & $27,76 €$ & $2137,90 €$ & $36,86 €$ \\
\hline TOTAL & & $\mathbf{9 7 0 3 , 8 6 €}$ & $\mathbf{1 6 7 , 3 1 €}$ \\
\hline
\end{tabular}

Table 2. Money potentially saved using teledentistry

\begin{tabular}{|c|c|c|}
\hline & For all appointments & By month \\
\hline Private practice & $1758,64 €$ & $30,32 €$ \\
\hline Public Hospital & $777,28 €$ & $13,40 €$ \\
\hline TOTAL & $\mathbf{2 5 3 5 , 9 2 €}$ & $\mathbf{4 3 , 7 2 €}$ \\
\hline
\end{tabular}


It is very impressive to state that only $3 \%$ of these 415 appointments have been realized under general anaesthesia and that $79 \%$ were realized on a classical pathway. A common way of thinking about oral treatment for disabled people is that they all need a general anaesthesia. This first element is very important. This point is identified on people with a lot of important incapacities (Figure 1) and with different types of treatment (Figure 3 ) it could be interesting to measure the same information on people with small incapacities in another type of facility.

About teledentistry, the University hospital of Montpellier has passed through a long history with this type of public health tool [9]. Telemedicine has already demonstrated to be very useful in different medical fields for a long time. Dentistry and oral medicine are late when compared with other medical domains but there is enough feedback all over the planet to assess that teledentistry is now ready to help everywhere [10].

Saving 104 appointments in 58 months is quite significant. It means almost 2 appointments $[1,8]$ per month.

We can also imagine that thanks to teledentistry we could diagnose earlier because the caregiver had the capacity to record videos every time he is suspicious. He didn't need to go to the dentist to show him what he saw on his own. Every transportation could be done when necessary and more effective. Because the dentist is already aware of what he has to do, we could suppose that he has prepared his equipment.

In case of disabled people, it is sometimes impossible for them to express themselves about the pain in their mouth or in another part of their body. Systematic teledentistry could detect every disease in the patients' mouth without organizing a transportation.

We saw that $32 \%$ of the appointments realized in a public hospital could be saved thanks to teledentistry. It is $9 \%$ more than for the appointment in private practices. It could be due to the fact that people going to the hospital because of their more severe incapacities are more difficult to be treated when conscious. How could it be imagined that every patient would accept to benefit of teledentistry? To record images for this activity, an intra oral camera has to be used and it needs to be accepted by the patient. It could be supposed that a patient with high incapacity wouldn't accept teledentistry and then should go to the dentist. But, the fact that a patient refuses an intra-oral camera could demonstrate that he is not able to be treated consciously and need to go directly to the hospital to be treated under general anaesthesia.

A last point on teledentistry: environment is crucial for disabled people, especially for those with mental incapacity. Teledentistry could be possibly more accepted than regular oral consultation for disabled people. Three points have to be mentioned:

- They are in their own facility, their own room, their known location;

- It is carried out by a caregiver who knows them better than their dentist does;

- The recording could be performed at the best time of the day for the patient acceptance and not when the dentist has time.
On the financial aspect, only $167,31 €$ per month could suffice to improve oral care in facility for disabled people with 41 patients. Moreover, this medico-economical study was a first step and didn't integrate some undirect impact as the cost for the facility of the caregiver accompanying only one patient to the dentist. This absence also creates disturbance in the organization because of one staff less to manage the 40 patients remaining in the facility.

According to our calculation, teledentistry could save $43,72 €$ per month to the facility. Of course, the number doesn't integrate the energy and stress saved for the patient. Every transportation is an effort for a high incapacity patient and for the people who are in charge of him/her.

The cost of teledentistry should be around $43,72 €$ per month for a facility to be economically viable.

\section{Conclusion}

Oral health in facilities for disabled people is neglected. Teledentistry seems a real opportunity to improve equality in access to care. The theoretical aspect is optimistic. The implementation of teledentistry and its evaluation in the next few years will be analysed to ensure that it is really efficient public health tool.

\section{References}

1. Dorin M, Moysan V, Cohen C, Collet C, Hennequin M (2006) Evaluation des besoins de santé bucco-dentaire des enfants et adolescents fréquentant un institut médicoéducatif ou un établissement pour enfants et adolescents polyhandicapés, en France. Prat Organ Soins 37: 14.

2. Nunn J (1987) The dental health of mentally and physically handicapped children - A review of litterature. Community Dent Health 4: 157-168. [Crossref]

3. Surabian S (2001) Developmental disabilities and understanding the needs of patients with mental retardation and Down Syndrome. J Calif Dent Assoc 29: 415-423. [Crossref]

4. Petrovic BB, Peric TO, Markovic DLJ, Bajkin BB, Petrovic D, et al. (2016) Unmet ora health needs among persons with intellectual disability. Res Dev Disabil 59: 370-377. [Crossref]

5. Turner S, Sweeney M, Kennedy C, Macpherson L (2007) The oral health of people with intellectual disability participating in the UK Special Olympics. J Intellect Disabil Res 52: 29-36. [Crossref]

6. Stanfield M, Stanfield M, Scully C, Davison MF, Porter S (2003) Oral healthcare of clients with learning disability: changes following relocation from hospital to community. Br Dent J 194: 271-277. [Crossref]

7. Kassebaum NJ, Smith AGC, Bernabé E, Fleming TD, Reynolds AE, et al. (2017) Global, regional, and national prevalence, incidence, and disability-adjusted life years for 195 countries, 1990-2015: A systematic analysis for the global burden of diseases, injuries, and risk factors. $J$ Dent Res 96: 380-387. [Crossref]

8. Folliguet M (2006) La santé bucco-dentaire des personnes handicapées. Direction Générale de la santé pp:51.

9. Giraudeau N, Valcarcel J, Tassery H, Levallois B, Cuisinier F, et al. (2014) Projet e-DENT : téléconsultation bucco-dentaire en EHPAD. Européenne en Télémédecine 3: 51-56.

10. Giraudeau N (2018) e-Health Care in dentistry and Oral Medicine: A clinician's guide. Springer pp: 154 .

11. Haute Autorité de Santé (2010) Stratégies de prévention de la carie dentaire. Paris: HAS pp: 180 .

Copyright: (C2019 Olivier R. This is an open-access article distributed under the terms of the Creative Commons Attribution License, which permits unrestricted use, distribution, and reproduction in any medium, provided the original author and source are credited. 